\title{
The effect of chronic exposure to dexamethasone on rocuronium-induced neuromuscular blockade and sugammadex reversal - in vivo study of rats
}

\author{
H. Y. Park ${ }^{1}$, S. K. Oh' ${ }^{2}$, H. Choi ${ }^{3}$, H. S. Yang ${ }^{4}$, J. In ${ }^{5}$
}

\section{Euroanaesthesia}

Department of Anesthesiology and Pain Medicine, Seoul National University Bundang Hospital - Sungnam (South Korea)

Department of Anesthesiology and Pain Medicine, Korea University Guro Hospital - Seoul (South Korea)

3Department of Anesthesiology and Pain Medicine, Seoul Paik Hospital - Seoul (South Korea),

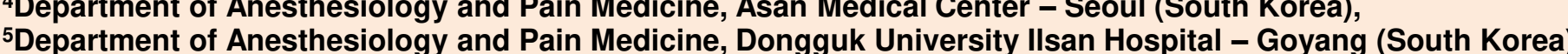

\begin{abstract}
"What we have found out"
Chronic exposure to dexamethasone does not significantly affect the sugammadex induced recovery of neuromuscular blockade.
\end{abstract}

\section{Background and Goal of Study}

Resistance to the actions of non-depolarizing neuromuscular blocking agents (NMBAs) are commonly associated with chronic exposure to glucocorticoid. It is known that chronic dexamethasone treatment induces alteration in immature subunits of nicotinic acetylcholine receptor (nAChR) and up-regulation of nAChR. Furthermore, up-regulation and spreading of acetylcholine receptors also induces the resistance to NMBAs. For this reason, we assumed that sugammadex induced recovery in subjects with long term treatment of dexamethasone is faster than in subjects without dexamethasone exposure. The goal of this study was to evaluate the recovery profile of the rocuroniuminduced neuromuscular blockade after the sugammadex administration.

\section{Materials and Methods}

Thirty adult male Sprague-Dawley rats ( 7 wk of age, $180-220 \mathrm{~g})$ were randomized into three groups $(n=10$ per group). The dexamethasone(Dexa) group received a daily intraperitoneal injection of Dexa $(500 \mu \mathrm{g} / \mathrm{kg})$ suspended in $0.9 \%$ normal saline for 15 days. The Control group received an equivalent volume of $0.9 \%$ normal saline daily for 15 days. The same amount of food consumed by the Dexa group was provided daily to the Pair-fed group. Rats were anaesthesized with Alfaxan ${ }^{\mathrm{TM}}$ and then tracheostomy and internal jugular catheterization were done. Both sciatic nerves were exposed and stimulated for twitch tension of the tibialis anterior muscle. Train-of-four (TOF) stimulations were applied supramaximally every 12 seconds. After administering rocuronium of $3.5 \mathrm{mg} / \mathrm{kg}$, the time to T2 (the second twitch of TOF) recovery was recorded. When T2 appeared, the sugammadex of $0.5 \mathrm{mg} / \mathrm{kg}$ was administered, then the recovery time from the T2 to the TOF ratio 0.9 was recorded.

\section{Results}

The time to T2 recovery was significantly shortened in the Dexa group compared with the Control group and the Pair-fed group (2.9 min [95\% confidence intervals, 2.2-3.6 min] vs. 5.0 [4.2- 5.8] and 5.1 [4.1-
$-6.1], P=0.001$, respectively) while no significant difference was observed between the Control group and the Pair-fed group $(P=0.996)$. There were no significant differences in the recovery time to TOF ratio to 0.9 among the groups (3.1 $\mathrm{min}$ [1.8-4.4 $\mathrm{min}$ ], 4.3 [2.6-5.9], and 4.0 [1.9-6.1], $P=0.531)$.

Table 1. Baseline comparison of animals in the study groups

\begin{tabular}{|c|c|c|c|c|}
\multicolumn{2}{|c|}{$\begin{array}{c}\text { Dexa } \\
(\mathrm{n}=10)\end{array}$} & $\begin{array}{c}\text { Control } \\
(\mathrm{n}=10)\end{array}$ & $\begin{array}{c}\text { Pair-fed } \\
(\mathrm{n}=10)\end{array}$ \\
\hline \multicolumn{2}{|c|}{ Weight of rat $(\mathrm{g})$} & $219 \pm 17^{*+}$ & $392 \pm 15$ & $322 \pm 18^{*}$ \\
\hline \multicolumn{2}{|c|}{ Body Temperature $\left({ }^{\circ} \mathrm{C}\right)$} & $37.6 \pm 0.2$ & $37.6 \pm 0.1$ & $37.5 \pm 0.2$ \\
\hline \multirow{2}{*}{$\begin{array}{c}\text { Tibialis } \\
\text { anterior } \\
\text { muscle }\end{array}$} & Weight $(\mathrm{mg})$ & $365 \pm 48^{*+}$ & $687 \pm 65$ & $565 \pm 21^{*}$ \\
\cline { 2 - 6 } & Length $(\mathrm{mm})$ & $19[18-21]$ & $22[21-22]$ & $21[21-21]$ \\
\cline { 2 - 6 } & $8[8-9]$ & $11[11-12]$ & $10[9-10]$
\end{tabular}

Data are presented as mean \pm standard deviation or median [interquantile range]. ${ }^{*} P<0.001$ vs. Control group. $+P<0.001$ vs. Pair-fed group.

One-way analysis of variance was performed followed by the Tukey post hoc test.

Table 2. Summary of data on the effects of chronic exposure to dexamethasone on duration of neuromuscular blockade and sugammadex reversal of rocuronium-induced neuromuscular blockade

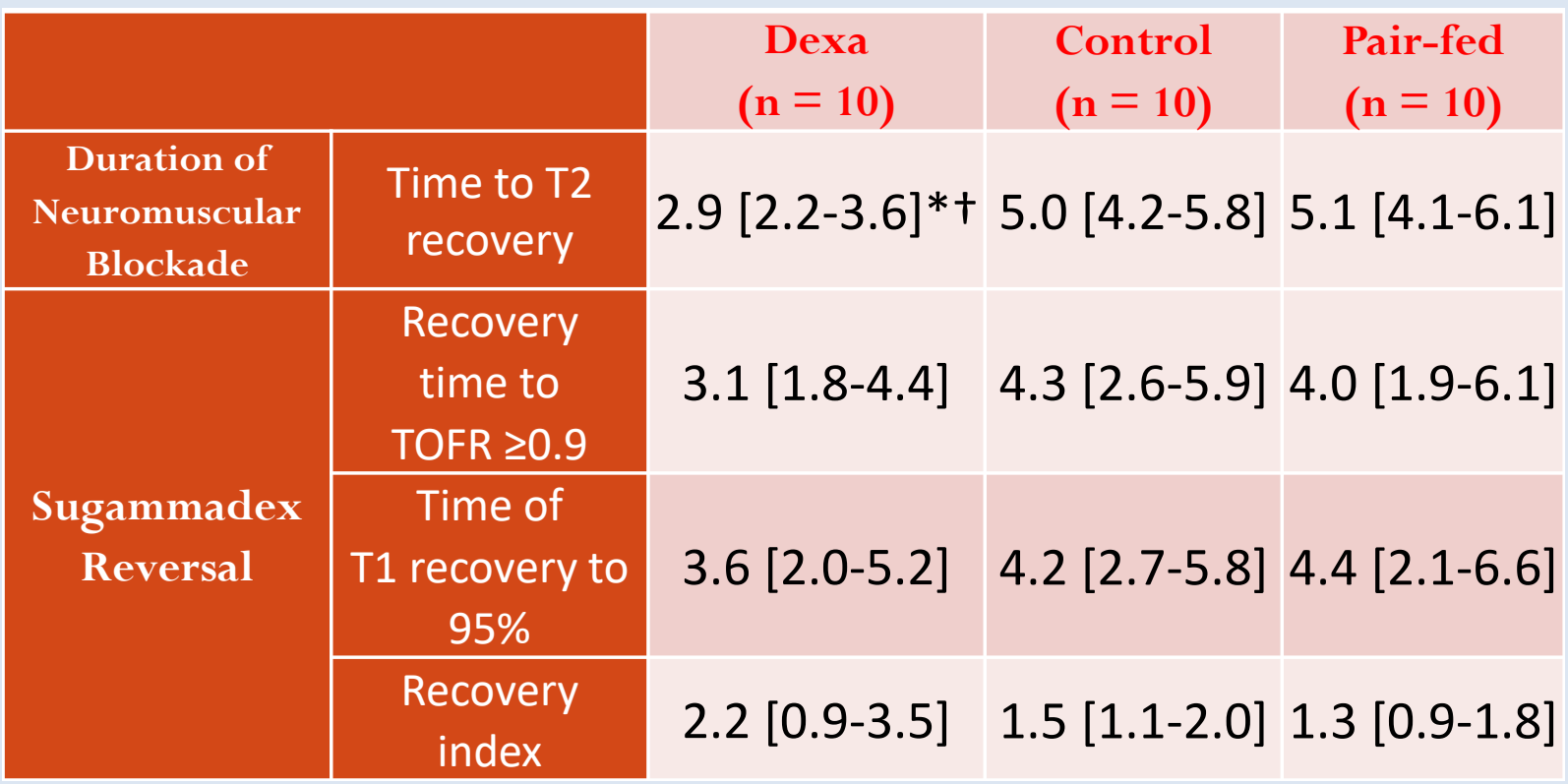

${ }^{*} P=0.001$ vs. Control group. $+P=0.001$ vs. Pair-fed group.

Time and recovery index are presented as minutes.

One-way analysis of variance was performed followed by the Tukey post hoc test. Data are presented as a mean [95\% confidence interval].

\section{Conclusions}

As we expected from the previous studies, resistance to rocuronium was observed with chronic exposure to dexamethasone. However, chronic exposure to dexamethasone did not significantly affect the sugammadex induced recovery of neuromuscular blockade.

\section{References}

1. Soltész S. Attenuation of a rocuronium-induced neuromuscular block in patients receiving prednisolone. Acta Anaesthesiol Scand 2009; 53: 443-8.

2. Chen D, Yang MR, Huang LN, Qiu YW, Li ST. Dexamethasone-induced hyposensitivity to rocuronium in rat diaphragm associated with muscle-fiber transformation. Mol Med Rep 2014; 9: 527-34. 